\title{
Besseres Heilen durch besseres Verstehen
}

K

aum etwas hat unsere Sicht auf allergische Erkrankungen in den letzten Jahrzehnten so verändert, wie das bessere Verständnis der immunologischen Grundlagen für deren Entstehung. Die Sachlage ist dabei nicht einfacher oder verständlicher geworden - im Gegenteil, immer mehr Zellen, Moleküle und Mediatoren kommen ins Spiel, viele behandelnden Allergologen werden inzwischen den Überblick in diesem Kosmos der Zytokine, Chemokine, Transkriptionsfaktoren, Kostimulatoren und „toll-like"-Rezeptoren verloren haben. Ein kleiner Versuch, etwas Ordnung in diese verwirrende Vielfalt zu bringen, soll dieses erste Themenheft der Sektion Immunologie der DGAKI sein. Da die Thematik sehr komplex ist und wir viele begeisterte Mitarbeiterinnen und Mitarbeiter in der Sektion haben, reicht diese erste Schwerpunktausgabe jedoch nicht aus, eine zweite wird im Mai folgen, welche sich mit den neuen Strategien zu Therapie und Prävention von Allergien und Asthma beschäftigen wird.

Dieses Heft konzentriert sich ganz auf die wesentlichen Zellarten, die bei der Ausbildung der allergischen Immunreaktionen beteiligt sind. Am Anfang einer jeden allergischen Kaskade steht die Aufnahme und Prozessierung des spezifischen Allergens. Knut Schäkel, „Viele haben den Überblick im Thilo Jakob und Joachim Kosmos der Zytokine, Chemo- Saloga stellen diesen wegweikine, Transkriptionsfaktoren, senden ersten Schritt in den Kostimulatoren und ,toll-like، Mittelpunkt ihrer BetrachRezeptoren verloren.“ tungen und erklären übersichtlich die wesentlichen Funktionen der dendritischen Zelle und ihren Einfluss auf die Differenzierung von T-Zellen zu „proallergischen “TH2-Zellen oder den anderen Spielarten der T-Zellen (S. 146).

Die verschiedenen Arten von T-Helferzellen stehen im Mittelpunkt des nächsten Artikels von Monika Raulf-Heimsoth und Philippe Stock. Ausgehend vom traditionellen TH1-/TH2-Konzept führen sie uns in die komplexer gewordene Differenzierung von T-Helferzellen ein und erklären, wie man anhand von Transkriptionsfaktoren und Zytokinmuster diese Subtypen unterscheiden kann (S. 152).

In einem weiteren Beitrag gehen die selben Autoren auf die Rolle der regulatorischen T-Zellen ein. Diese rücken immer mehr in den Mittelpunkt des Interesses, da ihnen eine wichtige Rolle bei der Aufrechterhaltung der immunologischen Balance zugeschrieben wird (S. 156).
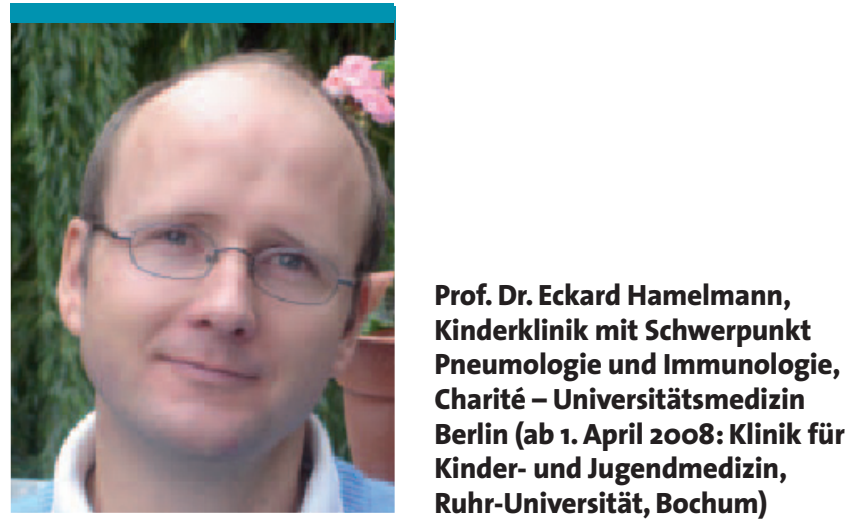

Margitta Worm und Martin Mempel halten dann die Fahne für die B-Zellen hoch, die manchmal etwas in den Schatten zu geraten drohen. Sie verschaffen uns einen sehr gelungenen Überblick über die Differenzierung von B-Zellen in verschiedene Subtypen und die molekularen Vorgänge beim Immunglobulin-Klassenwechsel bei der Bildung von IgE (S. 161).

Die folgenden beiden Artikel rücken dann Epithelzellen in den Vordergrund. Arnd Petersen und Robert Bals geben uns faszinierende neue Einblicke in die Rolle von Epithelzellen als wesentliche Schutzbarriere gegen das Eindringen von Allergenen (S. 165).

Von der Lunge zur Haut: Im Beitrag der Münchner Kolleginnen und Kollegen von GSF bzw. der Hautklinik der TU erfahren wir, dass Keratinozyten nicht nur Schutzfunktionen übernehmen, sondern auch wesentlich zwischen der angeborenen und adaptiven Immunität vermitteln können (S. 168).

Zugegeben, dieses Heft ist keine leichte Kost. Der Leser wird jedoch belohnt mit Aktualität, übersichtlicher Darstellung und grafischer Visualisierung der Zusammenhänge. Und wer die grundlegenden Mechanismen der allergischen Immunreaktion verstanden hat, wird sich sicher auf die darauf basierenden innovativen Strategien für Therapie und Prävention im zweiten Teil freuen!

In diesem Sinne wünsche ich im Namen der Sektion Immunologie der DGAKI allen Leserinnen und Lesern viel Vergnügen bei der Lektüre.

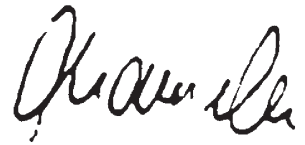

Prof. Dr. Eckard Hamelmann 